\title{
Epitaxy: Programmable Atom Equivalents Versus Atoms
}

\author{
Mary X. Wang, ${ }^{\&, \dagger, \ddagger}$ Soyoung E. Seo, ${ }^{\&, \S, \$}$ Paul A. Gabrys, ${ }^{\|}$Dagny Fleischman, ${ }^{\otimes}$ Byeongdu Lee, ${ }^{\#(C)}$

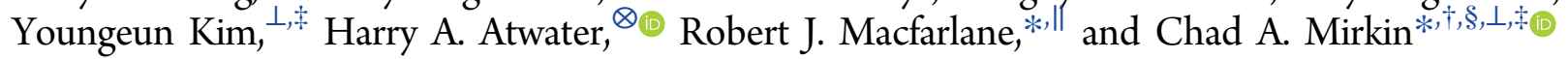

${ }^{\dagger}$ Department of Chemical and Biological Engineering, ${ }^{\star}$ International Institute for Nanotechnology, ${ }^{\S}$ Department of Chemistry, and ${ }^{\perp}$ Department of Materials Science and Engineering, Northwestern University, Evanston, Illinois 60208, United States

"Department of Materials Science and Engineering, Massachusetts Institute of Technology, 77 Massachusetts Avenue, Cambridge, Massachusetts 02139, United States

${ }^{\otimes_{T}}$ Thomas J. Watson Laboratories of Applied Physics, California Institute of Technology, 1200 East California Boulevard, Pasadena, California 91125, United States

${ }^{\#}$ X-ray Science Division, Argonne National Laboratory, 9700 South Cass Avenue, Argonne, Illinois 60439, United States

\section{Supporting Information}

ABSTRACT: The programmability of DNA makes it an attractive structure-directing ligand for the assembly of nanoparticle (NP) superlattices in a manner that mimics many aspects of atomic crystallization. However, the synthesis of multilayer single crystals of defined size remains a challenge. Though previous studies considered lattice mismatch as the major limiting factor for multilayer
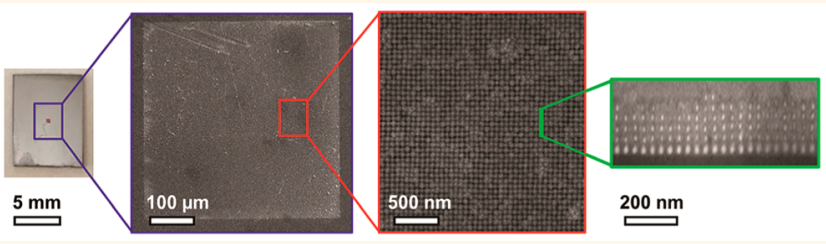
assembly, thin film growth depends on many interlinked variables. Here, a more comprehensive approach is taken to study fundamental elements, such as the growth temperature and the thermodynamics of interfacial energetics, to achieve epitaxial growth of NP thin films. Both surface morphology and internal thin film structure are examined to provide an understanding of particle attachment and reorganization during growth. Under equilibrium conditions, single crystalline, multilayer thin films can be synthesized over $500 \times 500 \mu \mathrm{m}^{2}$ areas on lithographically patterned templates, whereas deposition under kinetic conditions leads to the rapid growth of glassy films. Importantly, these superlattices follow the same patterns of crystal growth demonstrated in atomic thin film deposition, allowing these processes to be understood in the context of well-studied atomic epitaxy and enabling a nanoscale model to study fundamental crystallization processes. Through understanding the role of epitaxy as a driving force for NP assembly, we are able to realize 3D architectures of arbitrary domain geometry and size.

KEYWORDS: DNA, epitaxy, nanoparticles, self-assembly, thin film

1 he epitaxial deposition of thin films has been key to the semiconductor industry in its efforts to control material properties as a function of crystal structure. The transfer of order and orientation from a substrate to a deposited crystal is dependent upon many interlinked variables (e.g., interfacial chemical potential, crystal lattice parameters, defect stability) that vary as a function of both atomic composition and deposition protocol. ${ }^{1-3}$ As a result, significant effort has been expended to fully understand atomic epitaxy, leading to a wealth of information about thin film crystallization behavior. For nanoscale systems, many strategies have also been developed to assemble nanomaterials into thin films; ${ }^{4-8}$ however, these methods often lack the ability to precisely control the overall 3D structure of the resulting crystals (e.g., size, shape, and orientation).

Recent developments in nanoparticle (NP) assembly have shown that NPs functionalized with a dense monolayer of oligonucleotides can form ordered superlattice structures with programmable lattice parameters and crystallographic symmetries, and these building blocks exhibit many crystallization behaviors similar to those observed in atomic systems. ${ }^{9-11}$ Thus, these "programmable atom equivalents" (PAEs) hold promise for tailoring material structure at the nanoscale in a precise and controllable manner. In the context of PAE thin films, assembly on unpatterned surfaces has been demonstrated to produce rough, polycrystalline films with a lack of long-range order or alignment. ${ }^{12}$ Assembly of PAEs on patterned substrates has also been attempted but was limited to monolayers of single crystalline thin films, as the combination of both NP-substrate and NP-NP binding events significantly increases the complexity of multilayer epitaxial crystal

Received: September 29, 2016

Accepted: November 23, 2016

Published: December 5, 2016 
formation. ${ }^{4,13,14}$ In order to fully control thin film morphology in PAE superlattices, these complexities must be better understood via investigations into the thermodynamics of lattice growth as a function of different variables. The fundamental information gained from these comprehensive studies not only provides the opportunity to develop superlattice morphologies with complex $3 \mathrm{D}$ structures but also has the potential to provide insight into the process of atomic thin film epitaxy.

Like atomic systems, there are many design parameters that can affect multilayer epitaxy, such as factors inherent to the deposition protocol (e.g., thermal annealing temperature) and factors dictated by PAE design (e.g., DNA hybridization strength). ${ }^{10,12,15}$ However, unlike atomic epitaxy where only the deposition protocol can be modulated, parameters related to the individual PAE building blocks can be precisely controlled as a function of DNA, ${ }^{9,10,12}$ particle, $^{16-19}$ or substrate pattern design. ${ }^{13}$ Fully understanding how PAE epitaxy can be manipulated as a function of these variables will potentially yield single-crystal superlattices with controlled 3D geometries, allowing for the realization of materials possessing the desired optical, ${ }^{20-22}$ electronic, ${ }^{23}$ and magnetic responses. ${ }^{24}$ Here, we report a stepwise method for synthesizing large epitaxial thin films of PAEs up to 10 layers thick and $500 \mu \mathrm{m}$ wide and show that the corresponding growth process mimics atomic thin film epitaxy, allowing us to study epitaxy as a driving force for building a nanomaterial as it evolves from a $2 \mathrm{D}$ monolayer to a $3 \mathrm{D}$ crystal lattice.

\section{RESULTS AND DISCUSSION}

Epitaxial growth of NP superlattices was realized by depositing PAEs layer-by-layer onto lithographically defined substrates designed to resemble a continuous (100) plane of a bodycentered cubic (bcc) lattice (Scheme 1). Using standard

Scheme 1. Layer-by-Layer Assembly of PAE Superlattice Thin Films on a DNA-Functionalized Template

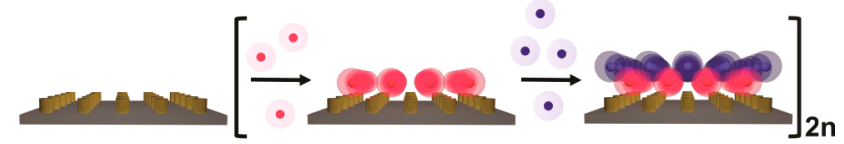

electron-beam lithography (EBL) techniques, $500 \mu \mathrm{m} \times 500$ $\mu \mathrm{m}$ arrays of gold posts were synthesized on a silicon wafer, such that post diameters and post-to-post distances were comparable to the PAE NP core diameters and the lattice parameter of the superlattice. These posts were functionalized with DNA and hybridized with complementary DNA linkers that presented a single-stranded recognition region to which PAEs could bind. Stepwise thin film growth was done via successive immersion of the template into suspensions of PAEs displaying a single-stranded recognition region complementary to that of the previous layer. They were then embedded in silica and characterized by synchrotron-based small-angle X-ray scattering (SAXS, Figure 1 middle) and grazing incidence small-angle X-ray scattering (GISAXS) to determine the overall degree of epitaxy. ${ }^{25}$ Lattices were also examined by scanning electron microscopy (SEM, Figure 1 top), which allowed for real-space imaging of superlattice surface morphology; focused ion beam milling was used to etch selected sections of the silicaembedded lattices, allowing for SEM characterization of the internal structure (FIB-SEM, Figure 1 bottom).
We first investigated the deposition of particles onto templated substrates via far-from-equilibrium conditions (i.e., low growth temperature) to understand the effectiveness of the EBL-patterned template itself as a driving force for multilayer epitaxy. When compared to atomic systems, this low temperature deposition is analogous to chemical bath deposition, where atoms rapidly precipitate from solution, resulting in disordered materials. ${ }^{26}$ Here, when conducting templated PAE deposition at $25{ }^{\circ} \mathrm{C}$, initial layers conform epitaxially to the substrate, but subsequent layers transition to a kinetically roughened, glasslike state (Figure 1a). This can be observed in the SAXS data, where a sample with two deposited layers shows diffraction spots (corresponding to aligned, single-crystal bcc lattices), while 5- and 10-layer films exhibit diffuse scattering, corresponding to disordered PAE aggregates. The relative degree of epitaxy $\left(X_{\mathrm{A}}\right)$ at each layer was determined by comparing the SAXS intensity of the spots from the (110) peak of the epitaxial PAEs and the intensity of the diffuse ring (Figure S1), where an $X_{\mathrm{A}}$ value of 1 indicates complete epitaxy of the PAEs; the value of $X_{\mathrm{A}}$ decays from 0.99 to 0.88 after 5 layers and to 0.65 at 10 layers (Figure 2a). This was corroborated by the FIB-SEM cross-sectional images, which showed that the first few layers of all samples are indeed epitaxial, up to a critical layer number of $\sim 4$, with PAEs above the critical layer adopting a kinetic, glassy state (Figure 1a, bottom, and Figure S2). This is a morphological transition commonly observed in atomic thin films, which exhibit temperature-dependent surface roughening when the adsorption rate is faster than the reorganization rate. ${ }^{2}$ Similarly, PAEs adsorbed at low temperature are stuck in kinetic traps, leading to an accumulation of defects in the film, which increases the surface area available for subsequent NPs to bind. This results in amorphous, rough films; the root-mean-squared roughness $\left(R_{\mathrm{RMS}}\right)$ increases by $140 \%$ from 2 - to 10 -layer deposited films (Table S2). The rapid, nonlinear increase in height, as measured by the mean $z$-distance of topmost NPs from the substrate, is further indication of nonequilibrium growth (Figure 2b). These data clearly show that the EBL template does serve as a strong driving force for epitaxy, but this driving force rapidly decays with increasing layer number when lattices are assembled at nonequilibrium conditions.

It is possible to reorganize thin films into more thermodynamically preferred configurations by adding thermal energy. This is done frequently in atomic systems to turn disordered or polycrystalline thin films (e.g., from sputter coating) into a film with a single-crystal orientation. This annealing process was mirrored in the PAE system by first depositing 5 and 10 layers at $25^{\circ} \mathrm{C}$ and then heating the sample slightly below the film's melting temperature $\left(T_{\mathrm{m}}\right.$, the temperature at which the superlattice dissociates). Interestingly, in the process of determining the films' $T_{\mathrm{m}}$, it was observed that they exhibit thickness-dependent melting point depression, analogous to atomic thin film systems (Figure S3). The thermal stability of the film, measured by monitoring lattice decomposition using SAXS, showed a concomitant increase with thickness due to the decreasing surface-to-volume ratio, as described by Lindemann's criterion and the Gibbs-Thomson relationship. Upon annealing the samples at $\left(T_{\mathrm{m}}-2\right){ }^{\circ} \mathrm{C}$, the 5-layer film became crystalline and epitaxial while retaining the same height and $R_{\mathrm{RMS}}$. On the other hand, the 10-layer sample became crystalline, but not epitaxial (Table S2 and Figure $1 \mathrm{~b}$ ). This is most likely due to the fact that the previously observed critical layer thickness for epitaxy is $\sim 4$, indicating that, in the 5- 


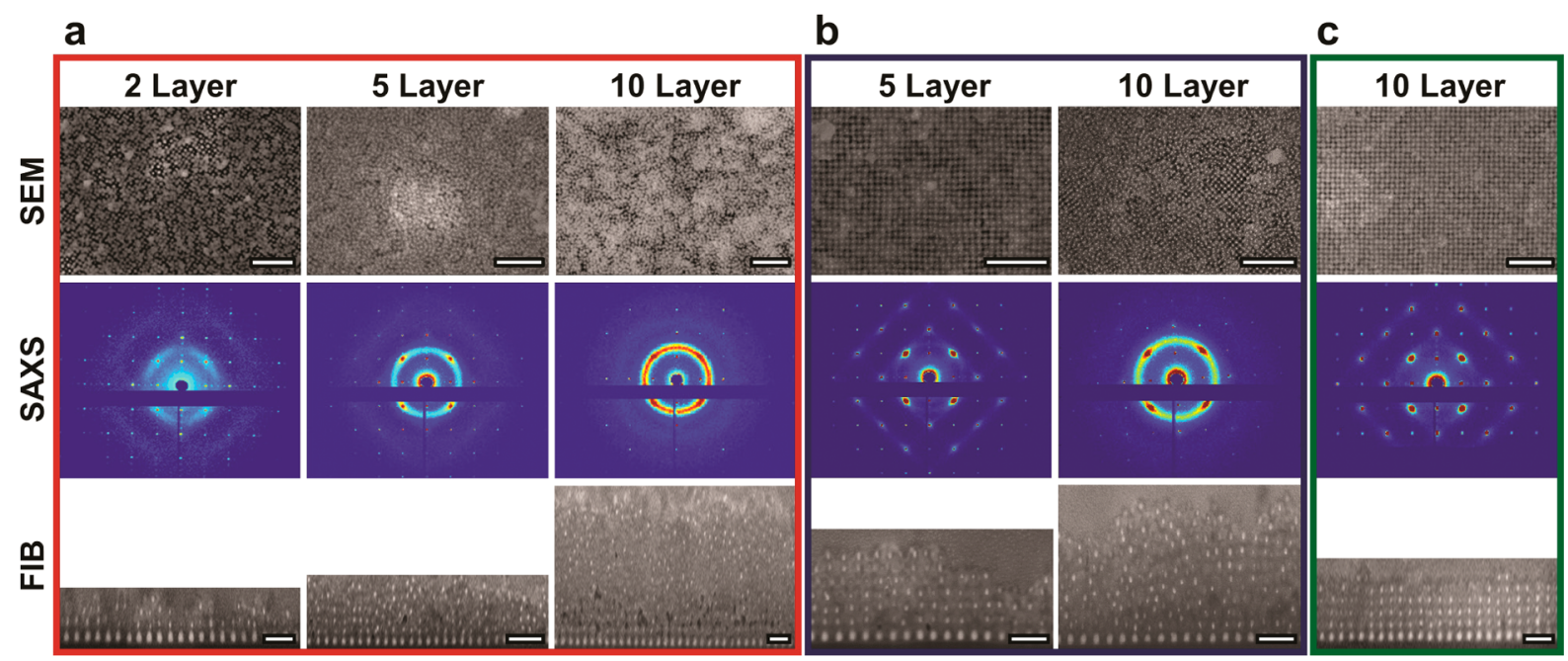

Figure 1. SEM, SAXS, and FIB-SEM characterization of DNA-NP thin films. (a) 2-, 5-, and 10-layer DNA-NP thin films assembled at $25{ }^{\circ} \mathrm{C}$ exhibit kinetic roughening and nonepitaxial growth beyond four layers of deposited PAEs. (b) 5- and 10-layer DNA-NP thin films assembled at $25{ }^{\circ} \mathrm{C}$ and thermally annealed after the full deposition process demonstrate enhanced ordering, but only the 5-layer sample is fully epitaxial since only PAEs that are close to the initial four epitaxial layers experience sufficient driving force to align with the patterned template. (c) 10layer DNA-NP thin film where each layer is assembled at an elevated temperature; this process produces smooth, crystalline thin films fully epitaxial with the patterned substrate. Scale bars for SEM and FIB-SEM are 500 and $200 \mathrm{~nm}$, respectively.
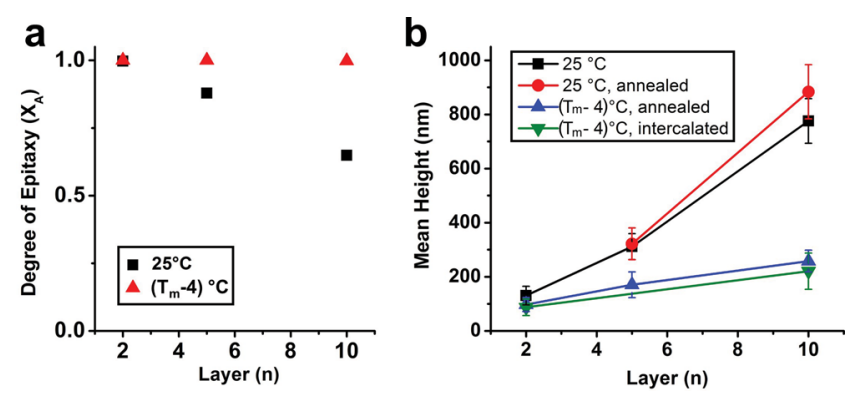

Figure 2. Quantitative characterization of films shows that depositing PAEs at near-equilibrium conditions induces (a) higher degree of epitaxy (as determined by SAXS) and (b) more controlled growth and a smoother film morphology (as determined from FIB-SEM).

layer sample, only the topmost layers of NPs were disordered. The differences in epitaxy for these two samples mirror previous findings for nonepitaxial PAE systems, in which postassembly annealing is capable of inducing crystallization but grain boundaries are difficult to remove once formed. ${ }^{15}$

The greatest degree of ordering in thin films can be achieved when the entire deposition process occurs under nearequilibrium conditions. To achieve this in atomic systems, molecular beam epitaxy is performed at high temperatures where deposition and desorption occur at equivalent rates, allowing each adatom to find its thermodynamic position in the monolayer before the next layer is introduced. To achieve this effect in the PAE system, each layer was deposited at an optimized growth temperature $\left(T_{\mathrm{m}}-4\right){ }^{\circ} \mathrm{C}$. With this method, nearly perfect Frank-van der Merwe (layer-by-layer) growth was observed, with films remaining epitaxial $\left(X_{\mathrm{A}}=0.99\right)$ far beyond the critical layer thickness of room-temperature growth (Figures 1c and 2a). Film cross-sections examined with FIBSEM show smooth surfaces with an absence of kinetic roughening; the $R_{\mathrm{RMS}}$ of the 10-layer film is $51 \%$ less than that of the equivalent film assembled at $25{ }^{\circ} \mathrm{C}$ (Table S2). Film height increases linearly with deposition layer, indicating that deposition occurred under equilibrium conditions (Figure $2 \mathrm{~b}$ ). Both GISAXS and SAXS confirmed that the film is well-ordered over a large area and nearly completely epitaxial with the patterned template (Figure 1c, middle, and Figure S4). Notably, a loss of radial broadening in the SAXS pattern, corroborated by FIB-SEM, indicates that as the film grows from 2 to 5 layers the stability of the PAE network increases to such a point that the particles become locked within a single domain. The appearance of thin lines of diffuse scattering between the peaks originates from the vibrational motion of the particles (Figures S5 and S6). This equilibrium growth condition was therefore able to create a crystalline film of well-defined and arbitrary crystal habit that is epitaxial over a domain of $500 \mu \mathrm{m}$ (Figure 3 and Figure S7).

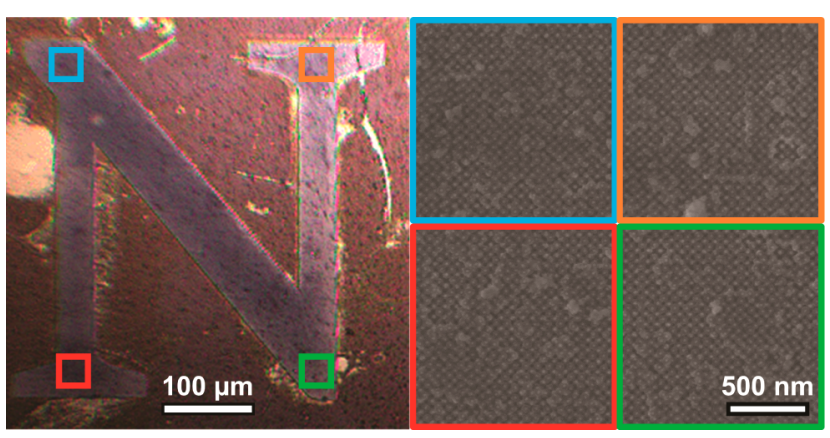

Figure 3. Optical image of DNA-functionalized NP thin film grown from a template exhibiting an arbitrary geometry. SEM images show that the thin film possesses the same crystallographic orientation across the entire structure.

Finally, an interesting aspect of this PAE system that does not have an atomic analogue is the ability to tune interfacial potential. In atomic crystals, chemical potentials between adatoms and the substrate are influenced by atomic identity and crystallographic symmetry. However, in PAEs, the chemical potential between NPs and the substrate can be tuned by adjusting DNA bond strength; we have recently demonstrated 
that this can be accomplished after assembly using DNA intercalators. $^{27,28}$ Here, these intercalators can be used to "staple" each PAE layer after deposition and annealing, thereby increasing their binding strength and preventing reorganization during subsequent cycles of growth. When this stapling method was employed, 10-layer thin films exhibited similarly high epitaxy $\left(X_{\mathrm{A}}=0.99\right)$ but $66 \%$ higher $R_{\mathrm{RMS}}$ than the nonintercalated counterpart, which can be attributed to defect immobilization by the intercalators (Figures S2 and S8 and Table S2). These data and the observed thickness-dependent melting point depression indicate that thermal annealing after each round of deposition induces reorganization not only in the topmost layer of PAEs but also in subsurface layers. This reorganization is critical for achieving perfect epitaxy, indicating the importance of being able to precisely modulate PAE binding strength during deposition.

\section{CONCLUSION}

In this work, we have determined that DNA-mediated NP crystallization follows similar thin film growth processes that are observed in atomic thin films, but importantly, PAEs offer a set of parameters distinct from atomic systems that can be independently tuned to control crystallization outcome. Unlike atomic systems, epitaxy can be controlled lithographically and through the choice of oligonucleotide bonding elements. These observations allow one to grow precisely defined crystalline NP architectures of arbitrary shape and size over thousands of $\mu \mathrm{m}^{2}$. Future studies will be able to take advantage of the tunable nature of the bonding interactions between the PAEs and the substrate to investigate how different parameters (DNA sequence, grafting density of DNA on PAEs) affect the epitaxial deposition process, laying the groundwork for making functional device architectures from crystalline NP networks.

\section{METHODS}

DNA Functionalization of Gold Nanoparticles. DNA functionalization on gold nanoparticles (AuNPs, $20 \mathrm{~nm}$ diameter from Ted Pella) was done using previously described methods. ${ }^{10,27}$ The $3^{\prime}$ propylmercaptan protecting group of the thiolated DNA (Table S1) was cleaved with $100 \mathrm{mM}$ dithiolthreitol (Sigma-Aldrich) for $1 \mathrm{~h}$, followed by desalting on a NAP5 size-exclusion column (GE Healthcare). The deprotected DNA was combined with colloidal AuNPs in a ratio of $6 \mathrm{nmol}$ of DNA per $1 \mathrm{~mL}$ of gold colloid. After a 30 min incubation, $1 \mathrm{wt} \%$ sodium dodecyl sulfate was added to bring the solution concentration to $0.01 \% . \mathrm{NaCl}(5 \mathrm{M})$ was added stepwise, followed by $10 \mathrm{~s}$ of sonication after each salt addition, until the final concentration of $0.5 \mathrm{M} \mathrm{NaCl}$ was achieved. The solution was then allowed to incubate in a shaker overnight to maximize the DNA loading (140 rpm, $\left.37{ }^{\circ} \mathrm{C}\right)$. Unbound DNA and excess salt were removed by four successive rounds of centrifugation and resuspension in nanopure water using a $100 \mathrm{kDa}$ filter centrifuge tube (Millipore) on a swinging bucket centrifuge $(2500 \mathrm{rpm}, 5 \mathrm{~min})$. After the last round of centrifugation, the DNA-NPs were concentrated down to the total volume of $500 \mu \mathrm{L}$. The concentrations of resulting DNA-NPs were determined using a Cary 5000 UV-vis-NIR spectrophotometer (Agilent) and known extinction coefficients from Ted Pella.

Substrate Preparation and Functionalization. Patterned Template Synthesis. Si wafers with native oxide $(\langle 100\rangle$, B doped, $10 \Omega \cdot \mathrm{cm}$ (Silicon Quest International)) were cleaned $(2 \mathrm{~min}$ acetone rinse, 2 min methanol rinse, drying under nitrogen) and baked for 2 min at $180{ }^{\circ} \mathrm{C}$. PMMA resist (495-A4) was spun onto the wafers $(3500 \mathrm{rpm}$ for $60 \mathrm{~s})$ and postbaked $\left(5 \mathrm{~min}\right.$ at $\left.180^{\circ} \mathrm{C}\right)$. Once they were cooled, 950-A2 PMMA resist was spun coat onto the coated wafers at $3500 \mathrm{rpm}$ for $1 \mathrm{~min}$ to create a bilayer and postbaked (5 min at 180 $\left.{ }^{\circ} \mathrm{C}\right)$. EBL was used to write the desired pattern with an optimized beam current $(500-700 \mathrm{pA})$ and dose range $\left(640-1500 \mu \mathrm{C} / \mathrm{cm}^{2}\right)$.
The substrates were developed in cold MIBK/IPA in a 1:3 ratio for 60 $\mathrm{s}$, briefly rinsed in IPA, and dried under nitrogen. The posts were then deposited using an electron-beam evaporator: $3 \mathrm{~nm}$ of $\mathrm{Cr}$ at a rate of $0.5 \AA / \mathrm{s}$ followed by $30 \mathrm{~nm}$ of $\mathrm{Au}$ at a rate of $1 \AA / \mathrm{s}$. Wafers were then diced into pieces with one pattern per chip. Liftoff was done in heated $\left(100-150{ }^{\circ} \mathrm{C}\right) \mathrm{PG}$ remover (Microchem); the chips were then rinsed (acetone followed by IPA) and finally dried under nitrogen.

Silanization of Patterned Templates. A hydrophobic hexamethyldisilazane (HMDS, Sigma-Aldrich) coating was employed to prevent nonspecific adsorption of DNA-NPs to the silicon chip, so that DNA-NP assembly occurred only on the DNA-functionalized Au posts of the template. This is a widely used vapor coating technique where the Si of HMDS reacts to form a strong bond with the oxidized silicon, creating a hydrophobic surface. Substrates were prebaked in a Vulcan 3-550 burnout furnace for $1 \mathrm{~h}$ at $150-200{ }^{\circ} \mathrm{C}$ to remove adsorbed water molecules. Silanization was performed by incubating the prebaked substrates in a sealed, dry chamber with a small open beaker containing $5 \mathrm{~mL}$ of HMDS and $5 \mathrm{~mL}$ of anhydrous hexane (Sigma-Aldrich) for $24 \mathrm{~h}$. After $24 \mathrm{~h}$, the substrates were rinsed and sonicated in water or ethanol for a few seconds.

Unpatterned Substrate Preparation. Unpatterned substrates were prepared by depositing a $2 \mathrm{~nm}$ Cr adhesion layer followed by $8 \mathrm{~nm}$ of $\mathrm{Au}$ on Si wafers using a PVD 75 E-beam evaporator (Kurt J. Lesker) at a base pressure of $5 \times 10^{-8}$ Torr. $\mathrm{Cr}$ and Au were evaporated at the rates of 0.3 and $0.5 \AA / \mathrm{s}$, respectively. These conditions yielded a smooth Au film, which is crucial for the crystalline DNA-NP thin film growth.

Substrate DNA Functionalization. DNA functionalization of substrates was performed by incubating each substrate in $2 \mathrm{~mL}$ Eppendorf tubes (Fisher Scientific) containing $5 \mu \mathrm{M}$ HS-A DNA solution diluted in buffer A $(0.5 \mathrm{M} \mathrm{NaCl}, 10 \mathrm{mM}$ phosphate buffered saline (PBS)) overnight. The propylmercaptan protecting group on the thiolated DNA was cleaved prior to the functionalization, as described above. The substrates were then washed three times in buffer A with vigorous agitation to remove unbound DNA and then hybridized with "linker" sequences (Table S1). "Linkers" consist of one complementary section that hybridizes to the thiolated DNA sequence, two double-stranded "duplexed" regions, and a short singlestranded sticky end. To prepare the duplexed linker stocks $(100 \mu \mathrm{M})$, "duplexer" strands were added to linkers A and B in a 2:1 molar ratio in $0.5 \mathrm{M} \mathrm{NaCl}$. The linkers were heated up to $70{ }^{\circ} \mathrm{C}$ for $5 \mathrm{~min}$ and cooled to room temperature over $4 \mathrm{~h}$ to achieve full hybridization. Duplexed linker stock solutions of $100 \mu \mathrm{M}$ concentration were made fresh every few weeks. Complementary linker (linker A) was hybridized to the DNA-functionalized substrate by incubating substrates in $0.5 \mu \mathrm{M}$ duplexed linker A solution at $0.5 \mathrm{M} \mathrm{NaCl}$ at $35{ }^{\circ} \mathrm{C}$ for $4 \mathrm{~h}$. Prior to layer-by-layer growth of DNA-NPs, the substrates were rinsed five times in buffer $\mathrm{A}$.

Layer-by-Layer DNA-Nanoparticle Superlattice Thin Film Assembly. Determining the Thin Film Annealing Temperature. To find the appropriate annealing temperature for the DNA-NP thin films, it is first necessary to determine the $T_{\mathrm{m}}$ of DNA-NP aggregates. Although the thermal melting and desorption behaviors are slightly different for solution-phase aggregates versus thin films, the $T_{\mathrm{m}}$ DNA$\mathrm{NP}$ aggregates can be used as a quick way to inform thin film assembly and annealing temperature. NP superlattice aggregates were prepared by mixing 0.5 pmol of DNA-functionalized $20 \mathrm{~nm}$ AuNPs (HS-A and HS-B) and 400 equiv per particle of each duplexed linker in a final concentration of $0.5 \mathrm{M} \mathrm{NaCl}$ at room temperature. Assembly was mediated by the complementary pendant "sticky ends" displayed on the linkers. After the sample was allowed to aggregate over 5-10 min, the thermal melting behavior was monitored using a Cary 5000 UVvis-NIR spectrophotometer. The extinction of the solution was monitored at 520 and $260 \mathrm{~nm}$ while the solution was heated from 25 to $60{ }^{\circ} \mathrm{C}$ at a ramp rate of $0.25{ }^{\circ} \mathrm{C} / \mathrm{min}$, and $T_{\mathrm{m}}$ was calculated from the point of inflection of the melting curve. Typically, reorganization and crystallization can be achieved by annealing a thin film superlattice at $2{ }^{\circ} \mathrm{C}$ below $T_{\mathrm{m}, \text { aggregate }}$ for $15 \mathrm{~min}$. This is experimentally determined by confirming the crystallinity of annealed DNA-NP thin films grown on unpatterned substrates using SEM (Figure S9). 
DNA-NP Superlattice Thin Film Assembly. A- and B-type DNANP assembly solutions were made by hybridizing each type of NP with its corresponding linker DNA (duplexed as described above) at 400 linkers per NP and incubated at $35{ }^{\circ} \mathrm{C}$ for $5 \mathrm{~min}$. The NPs were subsequently diluted to $1 \mathrm{nM}$ concentration $(0.5 \mathrm{M} \mathrm{NaCl}, 10 \mathrm{mM}$ PBS) and used for five layers of PAE assembly. DNA-NP superlattices were grown from the patterned substrates in a layer-by-layer fashion using four different growth conditions: (1) low-temperature growth, (1) low-temperature growth followed by annealing step, (3) elevated temperature growth, and (4) same conditions as 3 with an intercalation step after each annealing step. The intercalator solution was prepared by diluting $\left[\mathrm{Ru}\left(\right.\right.$ dipyrido $\left[3,2-a: 2^{\prime}, 3^{\prime}-c\right]$ phenazine $)\left(4,4^{\prime}\right.$ dimethyl-2,2'-bipyridine $\left.)_{2}\right] \mathrm{Cl}_{2}$ in $10 \mathrm{mM}$ PBS buffer. This compound was synthesized according to previously reported methods. ${ }^{29}$ For further details on the individual growth conditions, see Table S3. Layer-by-layer assembly was accomplished in the following way. Substrates functionalized with A-type DNA were incubated in a suspension of B-type DNA-NPs for $4 \mathrm{~h}$. Then the substrates were washed five times in buffer A and immersed in A-type DNAfunctionalized AuNP for $4 \mathrm{~h}$. This constituted two layers of DNANPs. For the annealing step, the substrate was incubated in buffer $A$ at an elevated temperature for $15 \mathrm{~min}$. This process was repeated until the desired number of layers was achieved. After the desired number of layers was reached, the samples were stored in buffer A at $25{ }^{\circ} \mathrm{C}$.

Silica Embedding. In order to transfer liquid-phase thin film superlattices to the solid state for characterization by SEM and GISAXS while preserving the structure, samples were embedded in silica using a sol-gel process. ${ }^{30}$ First, $3 \mu \mathrm{L}$ of $N$-(trimethoxysilyl)propyl- $N, N, N$-trimethylammonium chloride (TMSPA, Gelest, $50 \%$ in methanol) was added to the thin film superlattices in $1 \mathrm{~mL}$ of buffer $\mathrm{A}$ and left to fully associate with the DNA bonds within the superlattices for $30 \mathrm{~min}$ on an Eppendorf Thermomixer R $\left(1400 \mathrm{rpm}, 25^{\circ} \mathrm{C}\right)$. Then $5 \mu \mathrm{L}$ of triethoxysilane (Sigma-Aldrich) was added, and the sample was shaken for another $30 \mathrm{~min}$ before being removed. The samples were rinsed with running water and blown dry with $\mathrm{N}_{2}$. In the case of unsuccessful silica embedding, NPs will dissociate during the rinsing step. In order to prevent such failure, it is crucial to use dry, relatively fresh silane solutions (stored in a desiccator).

Small-Angle X-ray Scattering. All SAXS and GISAXS experiments were conducted at the 12ID-B station at the Advanced Photon Source (APS) at Argonne National Laboratory. The samples were probed using $14 \mathrm{keV}(0.8856 \AA) \mathrm{X}$-rays, and the sample-to-detector distance was calibrated with a silver behenate standard. The beam was collimated using two sets of slits, and a pinhole was used. The beam size was $\sim 200 \mu \mathrm{m} \times 50 \mu \mathrm{m}$. Scattered radiation was detected using a Pilatus $2 \mathrm{M}$ detector.

SAXS Experimental Conditions. Unembedded samples were probed using a vertical sample holder made from two coverslips that allowed a buffered environment to be maintained around the sample to preserve DNA hybridization. Embedded samples were mounted on a horizontal sample holder allowing for movement in the in-plane direction (normal to the beam). Sector averaging of diffraction patterns was used to determine the degree of epitaxy (Figure S1).

Grazing Incidence SAXS. Embedded samples were aligned to the beam on a sample positioning stage in the $x$ (parallel to the beam), $y, z$ (normal to the substrate), $\theta$ (rotation around the $y$-axis), and $\varphi$ (rotation around the $z$-axis) directions. The sample was centered in the X-ray beam by aligning the pattern to the center of the goniometer's $\theta$ and $\varphi$ rotations and by adjusting the sample height to be normal to the $\varphi$ rotation axis. Data were collected at incident angles of $0.1^{\circ}$. After alignment of the sample, scans were taken at several The sample was centered in the X-ray beam by aligning the pattern to the center of the goniometer's $\theta$ and $\varphi$ rotations and by adjusting the sample height to be normal to the $\varphi$ rotation axis. angles (rotation around the $z$-axis). In-depth information about GISAXS analysis is available from Senesi et al. ${ }^{12}$ and $\mathrm{Li}$ et al. ${ }^{25}$ GISAXS scattering patterns of 5-layer (Figure S4a) and 10-layer (Figure S4b) films were indexed to bcc crystals with (100) orientation corresponding to space group $I 4 / \mathrm{mmm}$ (\#139).
Focused Ion Beam-Scanning Electron Microscopy. After embedding samples in silica, a representative cross-section SEM image of each sample was obtained on a Helios Nanolab 600 dual beam focused ion beam milling system with a $52^{\circ}$ relative difference between the ion and electron beam. Scheme S1 shows the crosssection bcc crystallographic orientations obtained by FIB-SEM (Scheme S1). After a layer of titanium was deposited over the area of interest, a $15 \mu \mathrm{m} \times 1 \mu \mathrm{m}$ area, aligned lengthwise with the (100) followed by the (110) plane of the superlattice, was milled with a 93 pA $(30 \mathrm{kV})$ ion beam. Each cross-section was imaged with an $86 \mathrm{pA}$ (5 $\mathrm{kV}$ ) electron beam using the in-lens detector on the SEM without using the software's tilt correction. Postimage collection, SEM images of the cross sections were lengthened in the $y$-direction by the appropriate factor to account for the tilt. Data analysis on crosssections was done for entire $15 \mu \mathrm{m}$ cross-section; the larger image was subsequently cropped to a representative section and included in the figures for qualitative reference. The degree of epitaxy was determined using Photoshop and Matlab to track the positions of internal PAEs relative to the positions of the templated posts (Figures S2 and S5).

RMS Roughness and Mean Thickness Calculation. To calculate the thin film thickness and surface root-mean-square (RMS) roughness, the $15 \mu \mathrm{m}$ cross-section of (100) plane images was cropped into one image, where the posts met the substrate and the topmost PAEs were marked using Photoshop. Mean thickness was measured from the substrate surface to the center of the topmost PAE core and averaged over the entire cross-section (Table S2). Since FIBSEM images were taken at an angle, the images were adjusted for the tilt using Matlab prior to data processing. RMS roughness was calculated in its standard fashion: $R_{\mathrm{RMS}}=\sqrt{ }\left(\sum\left(y_{i}^{2}\right) / N\right)$, where $N$ is the number of PAEs on the thin film surface and $y_{i}=$ height-mean height.

\section{ASSOCIATED CONTENT}

S Supporting Information

The Supporting Information is available free of charge on the ACS Publications website at DOI: 10.1021/acsnano.6b06584.

Oligonucleotide sequences, FIB-SEM and SAXS analysis, and additional figures and tables (PDF)

\section{AUTHOR INFORMATION}

\section{Corresponding Authors}

*E-mail: rmacfarl@mit.edu.

*E-mail: chadnano@northwestern.edu.

ORCID

Byeongdu Lee: 0000-0003-2514-8805

Harry A. Atwater: 0000-0001-9435-0201

Chad A. Mirkin: 0000-0002-6634-7627

\section{Author Contributions}

${ }^{\&}$ M.X.W. and S.E.S. contributed equally.

\section{Notes}

The authors declare no competing financial interest.

\section{ACKNOWLEDGMENTS}

This work was supported by the following awards: AFOSR FA9550-11-1-0275 and FA9550-12-1-0280; the Department of Defense National Security Science and Engineering Faculty Fellowship N00014-15-1-0043; and the Center for Bio-Inspired Energy Science (CBES), an Energy Frontier Research Center funded by the U.S. Department of Energy (DOE), Office of Science, Basic Energy Sciences under award DE-SC00009890002. This work was also supported by the National Science Foundation's (NSF) MRSEC program (DMR-1121262) and made use of its Shared Facilities at the Materials Research Center of Northwestern University, specifically the EPIC 
facility of the NUANCE Center, which also receives support from the Soft and Hybrid Nanotechnology Experimental (SHyNE) Resource (NSF NNCI-1542205). X-ray experiments were carried out at beamline 12-ID-B at the Advanced Photon Source (APS), a U.S. DOE Office of Science User Facility operated by Argonne National Laboratory under Contract No. DE-AC02-06CH11357. EBL was performed at the Kavli Nanoscience Institute's shared instrumentation center. FIBSEM was performed at the Shared Experimental Facilities supported in part by the MRSEC Program of the NSF (DMR1419807). M.X.W. acknowledges support from the National Science Foundation Graduate Research Fellowship, a Ryan Fellowship, and the Northwestern University International Institute for Nanotechnology. S.E.S. acknowledges support from the Center for Bio-Inspired Energy Sciences Fellowship and the Northwestern University International Institute for Nanotechnology. Y.K. acknowledges support from a Ryan Fellowship and the Northwestern University International Institute for Nanotechnology.

\section{REFERENCES}

(1) Zhang, Z.; Lagally, M. G. Atomistic Processes in the Early Stages of Thin-Film Growth. Science 1997, 276, 377-383.

(2) Bartelt, M.; Evans, J. W. Transition to Multilayer Kinetic Roughening for Metal (100) Homoepitaxy. Phys. Rev. Lett. 1995, 75, $4250-4253$.

(3) Venables, J.; Spiller, G.; Hanbucken, M. Nucleation and Growth of Thin Films. Rep. Prog. Phys. 1984, 47, 399-459.

(4) Shevchenko, E. V.; Talapin, D. V.; Kotov, N. A.; O’Brien, S.; Murray, C. B. Structural Diversity in Binary Nanoparticle Superlattices. Nature 2006, 439, 55-59.

(5) Hua, F.; Shi, J.; Lvov, Y.; Cui, T. Patterning of Layer-by-Layer Self-Assembled Multiple Types of Nanoparticle Thin Films by Lithographic Technique. Nano Lett. 2002, 2, 1219-1222.

(6) Rupich, S. M.; Castro, F. C.; Irvine, W. T.; Talapin, D. V. Soft Epitaxy of Nanocrystal Superlattices. Nat. Commun. 2014, 5, 5045.

(7) Tao, A.; Kim, F.; Hess, C.; Goldberger, J.; He, R.; Sun, Y.; Xia, Y.; Yang, P. Langmuir-Blodgett Silver Nanowire Monolayers for Molecular Sensing Using Surface-Enhanced Raman Spectroscopy. Nano Lett. 2003, 3, 1229-1233.

(8) Kotov, N. A.; Dekany, I.; Fendler, J. H. Layer-by-Layer SelfAssembly of Polyelectrolyte-Semiconductor Nanoparticle Composite Films. J. Phys. Chem. 1995, 99, 13065-13069.

(9) Park, S. Y.; Lytton-Jean, A. K.; Lee, B.; Weigand, S.; Schatz, G. C.; Mirkin, C. A. DNA-Programmable Nanoparticle Crystallization. Nature 2008, 451, 553-556.

(10) Macfarlane, R. J.; Lee, B.; Jones, M. R.; Harris, N.; Schatz, G. C.; Mirkin, C. A. Nanoparticle Superlattice Engineering with DNA. Science 2011, 334, 204-208.

(11) O’Brien, M. N.; Jones, M. R.; Lee, B.; Mirkin, C. A. Anisotropic Nanoparticle Complementarity in DNA-Mediated Co-Crystallization. Nat. Mater. 2015, 14, 833-839.

(12) Senesi, A. J.; Eichelsdoerfer, D. J.; Macfarlane, R. J.; Jones, M. R.; Auyeung, E.; Lee, B.; Mirkin, C. A. Stepwise Evolution of DNAProgrammable Nanoparticle Superlattices. Angew. Chem., Int. Ed. 2013, 52, 6624-6628.

(13) Hellstrom, S. L.; Kim, Y.; Fakonas, J. S.; Senesi, A. J.; Macfarlane, R. J.; Mirkin, C. A.; Atwater, H. A. Epitaxial Growth of DNA-Assembled Nanoparticle Superlattices on Patterned Substrates. Nano Lett. 2013, 13, 6084-6090.

(14) Lalander, C. H.; Zheng, Y.; Dhuey, S.; Cabrini, S.; Bach, U. DNA-Directed Self-Assembly of Gold Nanoparticles onto Nanopatterned Surfaces: Controlled Placement of Individual Nanoparticles into Regular Arrays. ACS Nano 2010, 4, 6153-6161.

(15) Macfarlane, R. J.; Thaner, R. V.; Brown, K. A.; Zhang, J.; Lee, B.; Nguyen, S. T.; Mirkin, C. A. Importance of the DNA "Bond" in
Programmable Nanoparticle Crystallization. Proc. Natl. Acad. Sci. U. S. A. 2014, 111, 14995-15000.

(16) Zhang, C.; Macfarlane, R. J.; Young, K. L.; Choi, C. H. J.; Hao, L.; Auyeung, E.; Liu, G.; Zhou, X.; Mirkin, C. A. A General Approach to DNA-Programmable Atom Equivalents. Nat. Mater. 2013, 12, 741746.

(17) Zhang, Y.; Lu, F.; Yager, K. G.; van der Lelie, D.; Gang, O. A General Strategy for the DNA-Mediated Self-Assembly of Functional Nanoparticles into Heterogeneous Systems. Nat. Nanotechnol. 2013, 8, 865-872.

(18) Lee, J.-S.; Lytton-Jean, A. K.; Hurst, S. J.; Mirkin, C. A. Silver Nanoparticle-Oligonucleotide Conjugates Based on DNA with Triple Cyclic Disulfide Moieties. Nano Lett. 2007, 7, 2112-2115.

(19) Brodin, J. D.; Auyeung, E.; Mirkin, C. A. DNA-Mediated Engineering of Multicomponent Enzyme Crystals. Proc. Natl. Acad. Sci. U. S. A. 2015, 112, 4564-4569.

(20) Evanoff, D. D.; Chumanov, G. Synthesis and Optical Properties of Silver Nanoparticles and Arrays. ChemPhysChem 2005, 6, 12211231.

(21) Kelly, K. L.; Coronado, E.; Zhao, L. L.; Schatz, G. C. The Optical Properties of Metal Nanoparticles: The Influence of Size, Shape, and Dielectric Environment. J. Phys. Chem. B 2003, 107, 668677.

(22) Storhoff, J. J.; Lazarides, A. A.; Mucic, R. C.; Mirkin, C. A.; Letsinger, R. L.; Schatz, G. C. What Controls the Optical Properties of DNA-Linked Gold Nanoparticle Assemblies? J. Am. Chem. Soc. 2000, 122, 4640-4650.

(23) Choi, J.-H.; Wang, H.; Oh, S. J.; Paik, T.; Sung, P.; Sung, J.; Ye, X.; Zhao, T.; Diroll, B. T.; Murray, C. B. Exploiting the Colloidal Nanocrystal Library to Construct Electronic Devices. Science 2016, 352, 205-208.

(24) Jonsson, P. E. Superparamagnetism and Spin Glass Dynamics of Interacting Magnetic Nanoparticle Systems. Adv. Chem. Phys. 2003, $128,191-248$.

(25) Li, T.; Senesi, A. J.; Lee, B. Small Angle X-Ray Scattering for Nanoparticle Research. Chem. Rev. 2016, 116, 11128.

(26) Meyers, S. T.; Anderson, J. T.; Hung, C. M.; Thompson, J.; Wager, J. F.; Keszler, D. A. Aqueous Inorganic Inks for LowTemperature Fabrication of ZnO TFTs. J. Am. Chem. Soc. 2008, 130, 17603-17609.

(27) Seo, S. E.; Wang, M. X.; Shade, C. M.; Rouge, J. L.; Brown, K. A.; Mirkin, C. A. Modulating the Bond Strength of DNANanoparticle Superlattices. ACS Nano 2016, 10, 1771-1779.

(28) Pal, S.; Zhang, Y.; Kumar, S. K.; Gang, O. Dynamic Tuning of DNA-Nanoparticle Superlattices by Molecular Intercalation of Double Helix. J. Am. Chem. Soc. 2015, 137, 4030-4033.

(29) Shade, C. M.; Kennedy, R. D.; Rouge, J. L.; Rosen, M. S.; Wang, M. X.; Seo, S. E.; Clingerman, D. J.; Mirkin, C. A. Duplex-Selective Ruthenium-Based DNA Intercalators. Chem. - Eur. J. 2015, 21, 1098310987.

(30) Auyeung, E.; Macfarlane, R. J.; Choi, C. H. J.; Cutler, J. I.; Mirkin, C. A. Transitioning DNA-Engineered Nanoparticle Superlattices from Solution to the Solid State. Adv. Mater. 2012, 24, 51815186. 